(Aus dem physiologischen Institut der Universität Strassburg.)

\title{
Über die Wirkung schwacher elektrischer Doppelreize auf die quergestreifte und glatte Muskulatur des Frosches.
}

Von

stud. med. Siegfried Levinsohm.

(Mit 7 Textfiguren.)

Seit den grundlegenden Untersuchungen von $\mathrm{Helmholtz}{ }^{1}$ ) beschäftigen sich sehr viele Arbeiten mit der. Wirkung mehrfacher künstlicher Reize auf Muskeln und Nerven. Es hat sich gezeigt, dass im allgemeinen zwei oder mehrere gleich starke Reize wirksamer sind als einer allein. Diese Regel erleidet ein e Ausnahme: bekanntlich bleibt ein zweiter Reiz dann unwirksam, wenn er in sehr kleinem zeitlichen Abstand auf einen anderen folgt (Refralktärstadium). Dann ist also, was die sichtbare Wirkung anbetrifft, $\mathrm{I}+\mathrm{II}=\mathrm{I}$.

Von grosser theoretischer Wichtigkeit ist die Frage, ob mehrere Reize sich auch gegenseitig schwächen können, so dass z. B. bei zwei Reizen die Wirkung von I + II kleiner wird als die von I oder II. Über solche Interferenzen bei frequenter Reizung gibt es in der Literatur mehrere Angaben. Da diese Arbeit sich aber nur mit zwei Reizen beschäftigt, so soll auf die diesbezügliche Literatur, z. B. auf die Arbeiten von Fuld, Wedensky, F. B. Hofmann, F. W. Fröhlich hier nicht näher eingegangen werden.

Über Interferenzen zwischen zwei Reizen ist bisher nur wenig publiziert worden. Das hat wahrscheinlich folgenden Grund:

Wenn die Absehwächung einer Reizwirkung zur Beobachtung gelangen soll, so darf der Reiz nicht maximal (oder gar übermaximal) sein, denn eine geringere Verkleinerung eines Maximal-

1) H. Helmboltz, Monatsber. d. Berl. Akademie, 15. Juni 1855. 
reizes pflegt den Effekt nur wenig zu vermindern. Deshalb sind die zahlreichen Versuche über das Refraktärstadium nach Maximalreizen in dieser Hinsicht ohne Ergebnis. - Andererseits darf derjenige Reiz, dessen Abschwäehung untersucht werden soll, natürlich auch nicht unter der Schwelle liegen. Vielmehr muss er submaximal sein. Es ist leicht einzusehen, dass der andere entweder unterschwellig (subliminal) oder submaximal sein kann.

Wenn man das Problem so einengt, kommen nur wenige Arbeiten hier in Betracht.

Helmholtz ${ }^{1}$ ) gibt an, dass sich untermaximale Reize in jedem Intervall summieren. Dieser Forscher hat also keine „Subtraktion" beobachtet.

Ebenso findet sich in der neueren Arbeit von $\mathrm{Bazett}^{2}$ ) keine Angabe darüber, dass zwei Reize weniger wirken können als einer allein, trotzdem bei dieser Arbeit manchmal submaximale Reize zur Anwendung kommen.

Für das hier bearbeitete Problem ist die Arbeit von v. Kries und S ewa $11^{3}$ ) sehr wichtig. In dieser werden curarisierte Froschmuskeln durch $z$ wei submaximale Induktionsströme von gleicher oder entgegengesetzter Richtung gereizt. (Der zweite Fall soll unbesprochen bleiben, weil die Reizkathode dabei einmal mit der einen, das andere Mal mit der anderen Elektrode zusammenfällt, so dass die beiden Reize nicht dieselbe Stelle treffen.) Wenn der zweite Reiz stärker ist als der erste, so geben die Verfasser an, dass bei einem gewissen zeitlichen Intervall „die Höhe der Summierung zwar noch grösser ist als der Effekt des ersten Reizes, aber kleiner als der des zweiten Reizes allein". "Wenn der erste und der zweite Reiz," heisst es weiter, ngleich stark sind, so geht das Niveau der summierten Kontraktion auf den Wert der einzelnen herab, sinkt aber nicht (wenigstens nur ausnahmsweise und dann nur äusserst wenig) unter dieses Niveau. Ist der erste Reiz stärker, so sinkt die Summierungszuckung nicht unter das Niveau desselben herab, bleibt also stets höher als die Wirkung des zweiten Reizes allein." - Dann schreiben die Autoren, was für diese Arbeit von besonderem Interesse ist: "Es verdient vielleicht bemerkt zu werden, dass eine geringere Höhe beim Doppelreiz als

1) H. Helmholtz, I. c. S. 331 . Wissensch. Abh. Bd. 2 S. 884 .

2) H. C. Bazett, Journ. of physiol. vol. 36 p. $414-430$.

3) J. v. Kries und H. Sewall, Arch. f. (Anat. u.) Physiol. 1881 S. 66-77. 
bei einem der einzelnen sich (gleiche Richtung vorausgesetzt) nur dann zur Beobachtung bringen lässt, wenn beide Reize für sich wirksam sind, nicht dagegen, wenn einer von beiden unwirksam ist." In der Arbeit von v. Kries und Sewall ist also zum ersten Male nachgewiesen, dass ein schwacher Reiz die Wirkung eines stärkeren vorhergehenden oder folgenden vermindern kann:

In neuester Zeit hat Gildemeister) gezeigt, dass bei indirekter Muskelreizung die Wirkung eines Reizes auch dann vermindert wird, wenn ihm in bestimmtem zeitlichen Intervall ein unterschwelliger Reiz vorhergeht. Dieses Resultat könnte auch so ausgesprochen werden: Ein indirekt unterschwellig gereizter Muskel hat ein nicht sofort einsetzendes, rasch verschwindendes relatives Refraktärstadium, in dem er auf submaximale Reize weniger hohe Zuckungen ausführt als vorher und nachber.

Die vorliegende Arbeit sollte ermitteln, ob bei direkter Reizung der quergestreiften Muskulatur vielleicht Ähnliches zu finden wäre. Diese Frage ist zu bejahen, so dass der zuletzt zitierte Satz der Arbeit von v. Kries und Sewall nicht zu Recht besteht. Ausserdem habe ich meine Untersuchungen noch auf die atropinisierte Magenmuskulatur des Frosches ausgedehnt, wobei ich aber, wenn der erste Reiz unter der Schwelle lag, nicht zu klaren und eindeutigen Resultaten gekommen bin. Dagegen hat sich gezeigt, dass hier submaximale Reize durch einen nachfolgenden unterschwelligen abgeschwächt werden können.

\section{Methodik.}

Die beiden Reize waren Öffnungsinduktionsströme. Die Induktorien standen in beträchtlichem Abstande und senkrecht zueinander. Die primären Kreise waren vollständig voneinander getrennt, die sekundären waren so geschaltet, dass immer die gleichnamigen Pole der beiden sekundären Spiralen miteinander und mit einer Elektrode verbunden waren. Da auf diese Weise jede Spirale für die andere eine ziemlich gut leitende Nebenschliessung bildete, wurde zu jeder ein Widerstand von 1000 Ohm hinzugefügt.

Die Öffnungskontakte wurden von dem Plattenträger eines Federmyographions aufgeschlagen. Dieses wurde auf die hohe Kante

1) M. Gildemeister, Pflüger's Arch. Bd. 124 S. 447-461. Ferner: Beiträge zur. Physiologie und Pathologie, herausgeg. v. O. Weiss (Festschrif̂́t für L. Hermann) S. 53--58. Stuttgart 1908. 
gestellt, so dass die Führungsdrähte senkrecht standen. Dadurch bewegte sich der Plattenträger schneller und war weniger der Reibung auf den Drähten ausgesetzt. Seine Geschwindigkeit wurde durch Verzeichnung einer Stimmgabelkurve am Anfang und am Ende der Versuche festgestellt. Sie erwies sich als gut konstant und betrug in dem benutzten Bereich: $1 \mathrm{~mm}$ in 0,00045 Sek. Bei den Versuchen mit glatten Muskeln benutzte ich geringere Geschwindigkeiten; Näheres darüber ist auf S. 278 angegeben. Der eine Öffnungskontakt war verschiebbar, so dass das Intervall zwischen den beiden Reizen zwischen 0 und 0,0414 Sek. beliebig gewählt werden konnte. Gewöhnlich wurde er um ganze Millimeter verschoben; deshalb ist in den Figg. 1-4 und in der Tabelle 1 das Intervall von $1 \mathrm{~mm}=$ 0,00045 Sek. als Einheit gewählt worden. (Die Einheit der Fig. 6 und der Tabelle 2 ist auch $1 \mathrm{~mm}$, der aber dort 0,042 Sek. bedeutet.)

Die beiden zuerst benutzten sehr kleinen Induktorien waren einander gleich. Der Verlauf des Öffnungsstromes kann aus folgenden Daten beurteilt werden: Widerstand der sekundären Spirale 43,5 Ohm, Selbstinduktionskoeffizient 0,022 Henry ohne Kerne, 0,06 Henry mit Kernen aus dünnem Draht. Daraus lässt sich berechnen, dass im vorliegenden Falle, wo ausserdem noch mindestens $1000 \mathrm{Obm}$ im Kreise sind, die Öffnungsschläge in der Zeit, wo der Plattenträger die Strecke von $1 / 2 \mathrm{~mm}$ durcheilt, bis auf unmerkbare Reste verschwunden sind. Die Anwesenheit von Kernen aus dünnem Draht ändert daran nichts, wie Gildemeister ${ }^{1}$ ) nachgewiesen hat. Um ganz sicher zu gehen, dass der eine Strom schon vollständig abgelaufen war, wenn der andere anfing, habe ich den Stromverlauf noch experimentell bestimmt. Das geschah auf folgende Weise:

Der Öffnungskontakt I des Myographions wurde in den primären Kreis des einen Induktoriums eingeschaltet; in den sekundären Kreis desselben kam der Öffnungskontakt II und ein ballistisches Drehspulengalvanometer. Je längere Zeit jetzt zwischen der Öffnung von I und II vergeht, desto mehr von dem sekundären Strom kann auf das Galvanometer wirken, so dass man durch Variierung des Intervalls ein Bild des Stromverlaufs in der sekundären Spirale bekommt Durch Versuche dieser Art wurden die theoretisehen Schlüsse vollständig bestätigt.

1) M. Gildemeister, Über das Verschwinden der Magnetisierung. Ann. d. Physik (4) Bd. 23 S. 401-414. 
In einer anderen Versuchsreihe wurde das Galvanometer anstatt des Präparats eingeschaltet, wobei die Schaltung sich nicht von derjenigen unterschied, die am Anfange dieses Abschnitts beschrieben ist (die beiden Kontakte befanden sich also jetzt in den beiden primären Kreisen). Dann war immer dieselbe Ablenkung $\mathrm{zu}$ beobachten, gleichgültig ob die beiden Reize gleichzeitig oder mit zeitlichem Abstand erfolgten, vorausgesetzt dass dies Intervall klein im Vergleich zur Schwingungsdauer des Galvanometers war. Damit ist bewiesen, dass die später beschriebene "Subtraktion" nicht etwa einen rein physikalischen Grund hat.

$\mathrm{Zu}$ den Versuchen an glatten Muskeln dienten zwei grössere Induktorien mit Kernen, die nach denselben Methoden untersucht wurden. Hier brauchten die Öffnungsströme zu ihrem Ablauf beträchtlich längere Zeit, nämlich 0,009 Sek. Das kleinste Reizintervall (ausser Null) betrug aber bei diesen Versuchen 0,042 Sek., so dass die Verwendung dieser Ströme unbedenklich war.

Über die Zubereitung und Behandlung der Untersuchungsobjekte wird bei der Besprechung der Versuche das Nötige gesagt werden.

\section{Versuche.}

\section{A. Quergestreifte Muskulatur.}

Als Untersuchungsobjekte dienten überlebende Gastrocnemien (nur unvergiftet) und Sartorien (unvergiftet und curarisiert) von Esculenten und Temporarien. Der bequemeren Handhabung wegen verzichtete ich auf unpolarisierbare Elektroden, da nach den Untersuchungen von Gildemeister ${ }^{1}$ ) bei den Studien über Interferenz die Verwendung von Platinelektroden durchaus zulässig ist. Die Muskeln waren mit geringer Belastung (Gastrocnemien einige Gramm, Sartorien $0,7 \mathrm{~g}$ ) an leichten Schreibhebeln befestigt, welche die Zuckungen auf einer stillstehenden Kymographiontrommel aufzeichneten. Nach jedem Strich wurde diese ein wenig gedreht.

Die Versuche folgten einander im Abstande von 30 Sekunden, um die Ermüdung möglichst hintanzuhalten. Sie verliefen in folgender Weise: Zuerst wurde durch Veränderung des primären Stromes und des Rollenabstandes bei beiden Induktorien der Schwellenwert der Öffnungsströme aufgesucht. Dann wurde der Reiz I so weit ab-

1) M. Gildemeister, Beiträge zur Physiologie und Pathologie, herausgeg. vọn 0. Weiss (Festschrift für L. Hermann) S. 53—58. Stuttgart 1908. 
geschwächt, bis weder graphisch noch bei direkter Betrachtung des Muskels irgend eine Reaktion festzustellen war. (Wenn man auf die Lichtreflexe, besonders in der Nähe der Kathode, achtet, so macht die Entscheidung, ob ein Reiz über- oder unterschwellig ist, gar keine Schwierigkeit.) Der Reiz II wurde dagegen so weit verstärkt; bis die Zuckung $1 / 3$ bis $1 / 2$ der maximalen Höhe hatte ${ }^{1}$ ). Dann folgten einander die fünf Teilversuche: $\alpha$. Reiz I allein; $\beta$. Reiz II allein; $\gamma$. Reiz I + Reiz II in bestimmtem, auf der Millimeterskala des verschiebbaren Kontaktes ablesbarem zeitlichen Abstande; $\delta$. derselbe Teilversuch $\gamma$ wiederholt; $\varepsilon$. zur Kontrolle nochmals Teilversuch $\beta$. - Wenn eine solche Versuchsserie beendigt war, so folgte sofort eine andere mit verändertem Intervall, wobèi ịch gewöhnlich um ganze Millimeter fortschritt $(1 \mathrm{~mm}=0,00045$ Sek.).

1. Unvergiftete Gastrocnemien. Die ersten Versuche sind nur als Vorversuche zu betrachten, bei denen ich mir die nötige Fertigkeit in der Handhabung des Instrumentariums erwarb. Die Muskeln wurden teils unbedeckt untersucht, wobei sie von Zeit zu Zeit mit Locke'scher Lösung $(\mathrm{NaCl} 0,6, \mathrm{KCl} 0,01, \mathrm{CaCl} 0,02$ in 100 Wasser) bepinselt wurden, teils nach dem Vorgange von v. Kries und Sewall unter ihrer eigenen Hautbedeckung, teils in einer feuchten Kammer. Der eine Platindraht wurde an der Sehne befestigt, der andere an der dicksten Stelle in das Muskelfleisch hineingestochen. Dieser primitiven Methodik entsprechend waren die Resultate ziemlich schwankend, aber in 6 unter 17 Fällen deutlich positiv, d. h. der Doppelreiz wirkte bei einem gewissen Intervall weniger als der Einzelreiz. Irrtümlich benutzte jch zuerst eine falsch zusammengesetzte Locke'sche Lösung (zu viel $\mathrm{K}$ und $\mathrm{Ca}$ ). Das mag auch zu dem ungleichmässigen Resultat beigetragen haben.

Einige Beobachtungen will ich noch erwähnen. Das Subtraktionsstadium war manchmal deutlicher, wenn die Sehne zur Anode gemacht wurde. Ferner schien es von günstigem Einfluss zu sein, wenn der mit den Kathoden der Induktorien verbundene Platindraht nur wenige Muskelfasern schlingenförmig umfasste:

Von technischen Kunstgriffen, die sich als sehr nützlich für die Erlangung guter und ausdanernder Präparate erwiesen, mögen noeb folgende angeführt werden: Bei der Tötung des Frosches ist es gut, in bekannter

1) Die besten Resultate geben solche Muskeln, bei denen kleine Änderungen der Reizstärke grossen Anderungen der Zúckungshöhe entsprechien. 
Art mit der Durchschneidung der Wirbelsäule dicht über dem Becken anzufangen, damit die Beinmuskulatur dureh das Ausbohren usw. nicht unnütz erregt wird. Ferner scheinen Frösche, die in Holzgefässen aufbewahrt sind, viel ausdauernder zu sein als solche, die in Zinkgefässen gesessen haben. Als ich diese Vorsichtsmaassregeln beachtete, gaben gegen das Ende meiner Untersuchungen., die vom November bis März dauerten, fast alle Versuche ein positives Resultat, während das, wie schon erwähnt, am Anfange anders war.

\section{Tabelle 1.}

Resultate der Doppelreizung von quergestreiften Muskeln. + Summation $(++\operatorname{sehr}$ stark), - Subtraktion (- sehr stark), = Indifferenz. Erster Reiz unterschwellig, zweiter submaximal, nur beim Intervall -1 umgekehrt. $\downarrow$ absteigender, $\uparrow$ aufsteigender Strom.

\begin{tabular}{|c|c|c|c|c|c|c|c|c|c|c|c|c|c|c|c|c|c|}
\hline \multirow{2}{*}{$\mathrm{Nr}}$. & \multirow{2}{*}{$\begin{array}{c}\text { Versuchs- } \\
\text { objekt }\end{array}$} & \multicolumn{16}{|c|}{$\begin{array}{c}\text { Intervalle zwischen subliminalem und submaximalem Reiz. } \\
\text { Einheit } 0,00045 \text { Sek. }\end{array}$} \\
\hline & & -1 & 0 & $|0,5|$ & 1 & $|1,5|$ & 2 & 4 & 6 & 18 & 10 & 15 & 20 & 25 & $\mid 30$ & 40 & 50 \\
\hline $\begin{array}{r}5 \\
6 \\
7 \\
8 \\
9 \\
16\end{array}$ & $\begin{array}{l}\text { Gastro- } \\
\text { cnemius } \\
\text { unvergiftet }\end{array}$ & & $\mid \begin{array}{c}++ \\
+ \\
+ \\
+\end{array}$ & & $\begin{array}{l} \pm \\
= \\
+\end{array}$ & & $\begin{array}{l} \pm \\
- \\
-\end{array}$ & $\begin{array}{l}- \\
= \\
= \\
-\end{array}$ & $1-$ & $=$ & $=$ & $=$ & $\begin{array}{l}= \\
=\end{array}$ & $=$ & $=$ & & $=$ \\
\hline $\begin{array}{r}20 \\
25 \\
26 \\
27\left\{\begin{array}{l}\downarrow \\
\uparrow \\
\downarrow\end{array}\right. \\
\end{array}$ & \begin{tabular}{|cc} 
Sartorius \\
unver- & $\mathrm{a}$ \\
giftet & $\mathrm{b}$ \\
& $\mathbf{c}$ \\
\end{tabular} & + & $\begin{array}{l}++ \\
+ \\
+ \\
+ \\
+\end{array}$ & $=$ & + & $=$ & -- & $\mid \begin{array}{c}- \\
+ \\
=\end{array}$ & & - & $\begin{array}{l}!= \\
= \\
=\end{array}$ & & $=$ & & $=$ & & \\
\hline $\begin{array}{l}28 \\
29 \\
31 \\
\end{array}$ & $\begin{array}{c}\text { Sartorius } \\
\text { unvollständig } \\
\text { curarisiert }\end{array}$ & & $\begin{array}{l}++ \\
++ \\
++\end{array}$ & & & & $\begin{array}{l}-- \\
--\end{array}$ & $\begin{array}{l}-- \\
-- \\
-\end{array}$ & & & $=$ & & $\underline{\bar{z}}$ & & & & \\
\hline 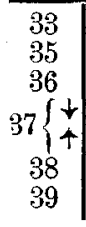 & $\begin{array}{c}\text { Sartorius } \\
\text { voll- } \\
\text { ständig } \\
\text { curarisiert }\end{array}$ & + & $\begin{array}{l}+ \\
+ \\
+ \\
++ \\
+ \\
+ \\
+\end{array}$ & & $\begin{array}{l}+ \\
+ \\
+\end{array}$ & & $\begin{array}{l}-- \\
=- \\
=- \\
= \\
-\end{array}$ & $\begin{array}{l}-- \\
- \\
-- \\
- \\
--\end{array}$ & $=$ & $=$ & $=$ & & $\begin{array}{l}= \\
= \\
=? \\
=\end{array}$ & & $\begin{array}{l}= \\
=\end{array}$ & $=$ & $=$ \\
\hline
\end{tabular}

Die Resultate der sechs positiven Versuche sind aus dem ersten Teil der Tabelle $1 \mathrm{zu}$ ersehen. Es braucht wohl nicht besonders gesagt zu werden, dass die Bezeichnung "Summation" gewählt worden ist, wenn der Doppelreiz eine höhere Zuckung ergibt als der einzelne, dass bei "Subtraktion" das Gegenteil stattfindet, und dass "Indifferenz" die Gleichheit beider Zuckungen bedeutet. Subtraktion 
findet hier statt bei einem Intervall von ungefähr zwei bis vier Einheiten, d. h. 0,0009 bis 0,0018 Sek., einmal noch etwas länger. Es verdient hervorgehoben zu werden, was auch die folgenden Versuche lehren, dass $\mathrm{Summation}$ bei quergestreiften Muskeln niemals $\mathrm{zu}$ beobachten war, wenn das Intervall zwischen beiden Reizen den Wert von 0,0018 Sek. überstieg. Glatte Muskeln verhalten sich anders. (Vgl. S. 279 Mitte.)

Um das Gesagte zu verdeutlichen, ist der Versuch 9 in Fig. 1 abg ebildet.

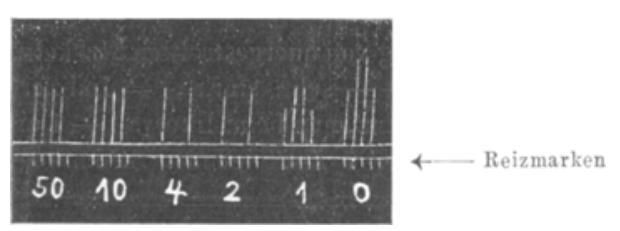

Fig. 1. Versuch 9. Zuckungen eines nicht curarisierten Gastrocnemius bei Doppelreizung. Von rechts nach links zu lesen. Die Zahlen bedeuten Intervalle zwischen Reiz I (unterschwellig) und Reiz II (submaximal); Einbeit 0,00045 Sek. Jede Gruppe besteht aus fünf Teilversuchen : $\alpha$. Reiz I (keine Zuckung); $\beta$. Reiz II; $\gamma$. Reiz I + Reiz II; $\delta$. wie $\gamma ; \varepsilon$. wie $\beta$. Summation bei $\theta$ und 1 , Subtraktion bei 2 and 4 , Indifferenz bei 10 und 50 .

2. Unvergiftete Sartorien. Da bei der beschriebenen Technik wahrscheinlich immer nur einzelne Fasern des Gastrocnemius gereizt wurden, und da sich dieser Muskel überhaupt wegen seiner unregelmässigen Faserung zu direkten Reizversuchen schlecht eignet, ging ich zu der Benutzung des Sartorius über. Zuerst hatte ich hier keinen Erfolg; erst als ich alle oben beschriebenen Vorsichtsmaassregeln beobachtete und ausserdem zum Zweck des sauberen Präparierens eine stereoskopische Lupe benutzte, kam es fast gar nicht mebr vor, dass ein Versuch negativ ausfiel.

Die beiden Endsehnen wurden sorgfältig geschont. Um die Erregbarkeit möglichst gut -zu konservieren, wurde der Muskel ganz in Locke'sche Lösung versenkt. Die "nasse Kammer" bestand aus einer Glasröhre von $55 \mathrm{~mm}$ Länge und $19 \mathrm{~mm}$ Weite. Unten war sie mit einem Gummistopfen versehlossen, durch den ein kurzes Glasröhrchen bindurchgesteckt war. Dieses trug wieder an seinem dem Innern der Kammer zugekehrten Ende ein Stück Kork, auf dem die Beckensehne des Muskels mit einer Nadel festgesteckt wurde. Die stromführenden Platindrähte, voneinander durch Paraffin isoliert, liefen durch das Glasröhrchen und durchbohrten den Kork, der mit Paraffin getränkt war. Der eine berührte den Muskel 
möglichst dicht an der Beckensehne, der andere $2 \mathrm{~mm}$ weiter distal. An die Kniesehne wurde ein feiner Seidenfaden geknüpft, dann wurde die Kammer mit der Öffnung nach oben festgeklemmt, mit Lockescher Lösung gefüllt und schliesslich der Faden mit dem Schreibhebel verbunden. An einem dauernd eingetauchten Thermometer konnte die Temperatur der Flüssigkeit, die zwischen 14 und $16^{\circ} \mathrm{C}$. schwankte, abgelesen werden.

Die Induktionsströme hatten meistens absteigende Richtung; wo es anders war (Versuch 27 und 37), ist es in der Tabelle besonders erwähnt.

Die Resultate sind aus dem zweiten Teil der Tabelle $1 \mathrm{zu}$ ersehen. Auch hier ist etwa in demselben Bereiche wie beim Gastrocnemius deutliche Subtraktion zu erkennen.

Der Versuch Nr. 27, von dem der erste Teil in Fig. 2 reproduziert ist, verdient besondere Beachtung. Hier zeigte sich mit aller Sicherheit eine Beeinflussung des Subtraktionsbereichs von der Stromrichtung. Das Intervall 1 fiel bei absteigendem Strom schon in den Subtraktionsbereich, während es bei aufsteigendem noch dem initialen Summationsbezirk angehörte. Dass hier kein Zufall obwaltete, lehrt der Kontrollversuch $(27 \mathrm{c})$. Vielleicht fiel im ersten Falle die Kathode in die Gegend der Nervenendigungen, wo nach Keith Lucas ${ }^{1}$ ) zwei, verschiedenen Gesetzen folgende, erregbare Substanzen vorhanden sind.

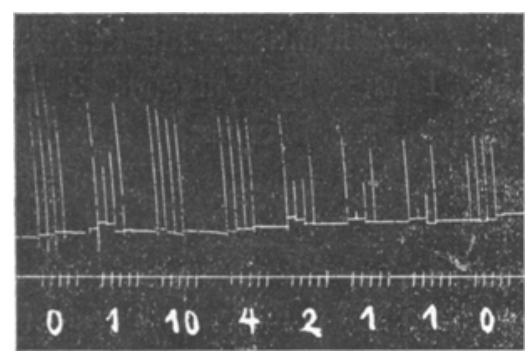

Fig. 2. Versuch 27 a. Sartorius, nicht curarisiert. Von rechts nach links zu lesen. Erklärung siehe Fig. 1. Steigende Erregbarkeit.

3. Curarisierte Sartorien. Das Ergebnis der oben beschriebenen Versuche deckt sich ungefähr mit den von Gildemeister am Nervmuskelpräparate erhobenen Befunden. Das konnte

1) Keith Lucas, Journ. of physiol. vol, 35 p. $103-114$. 
daran liegen, dass auch hier die im Muskel verlaufenden Nerven gereizt wurden, obgleich diese Erklärung für die Sartoriusversuche nicht sehr wahrscheinlich ist. Denn die Kathode lag dort, wo nach Kühne keine Nerven vorhanden sind. Um die Frage, ob auch die Muskelsubstanz ein Subtraktionsstadium besitzt, zu entscheiden, benutzte ich durch Curare entnervte Muskeln. Das Gift wurde in $1 \%$ iger Lösung (gewöhnlich $1,5 \mathrm{ccm}$ ) in den Rückenlymphsack eingespritzt und das Präparat erst dann angefertigt, wenn das Tier vollständig bewegungslos geworden war. Bei der Präparation untersuchte ich durch elektrische und mechanische Reizung des N. ischiadicus, ob das Gift die indirekte Erregbarkeit der. Muskeln vollständig aufgehoben hatte. Wenn das der Fall war, so wurde das Tier als „vollständig curarisiert" hezeichnet, wenn aber noch die geringste Reaktion zu erzielen war, als „unvollständig curarisiert". Als ich frisches Curare von Grübler-Leipzig benutzte, kam letzteres nicht mebr vor.

Es ist noch zu erwähnen, dass die vollständig vergifteten Muskeln in $1 / 10 \%$ iger Curare-Locke-Lösung untersucht wurden, damit das Gift nicht etwa ausgewaschen werden konnte. (Nach den Angaben von Keith Lucas ${ }^{1}$ ) hätte schon 1/10 dieser Konzentration zu dem beabsichtigten Zwecke genügt.) Eine stärkere Lösung habe ich deshalb nicht benutzt, weil Curare nach der Arbeit von Min es ${ }^{2}$ ) sehr reich an löslichen anorganischen Substanzen ist.

Die Resultate sind im dritten und vierten Teil der Tabelle 1 dargestellt. Sie sind ganz identisch mit den früheren (Intervall $0 \mathrm{~mm}^{3}$ ): Summation; $1 \mathrm{~mm}$ : schwankend; 2 und $4 \mathrm{~mm}$ : Subtraktion; $10 \mathrm{~mm}$ und mehr: Indifferenz.). Der Muskel verhält sich nach der Curarisierung also auscheinend genau so wie vorher: die Lage des Subtraktionsbezirkes wird dadurch nicht merklich verändert.

Die Figg. 3 und 4 führen die Versuche 29 (unvollständige Vergiftung) und 35 (vollständige Vergiftung) vor Augen. Letzterer zeigt deutlich, dass die Subtraktion sich auf einen ganz kleinen Bereich beschränken kann (vgl. Intervall 4 mit 2 und 6 ). Noch auffälliger ist dies bei Versuch 25 (s. die Tabelle).

Nach den Ergebnissen dieses Abschnittes ist die nebenstehende Fig. 5 gezeichnet worden. Die Einzelheiten sind aus der Legende

1) Keith Lucas, Journ. of physiol. vol. 36 p. 126.

2) Mines, Journ. of physiol. vol. 37 p. 416.

3) $1 \mathrm{~mm}=0,00045$. Sek. 


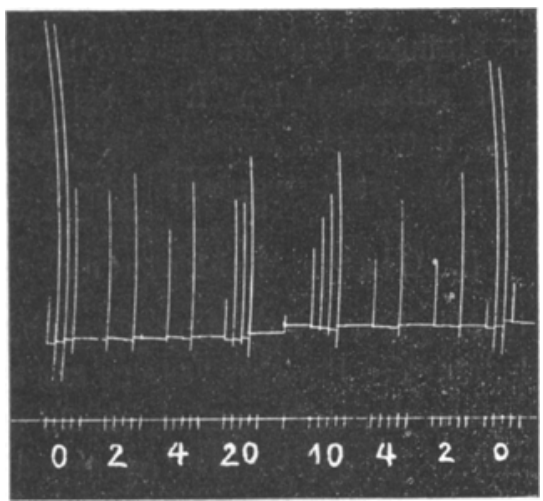

Fig. 3. Versuch 29. Sartorius, unvollständig curarisiert. Von rechts nach links zu lesen. Erklärung siehe Fig. 1. Anscheinend starke Ermüdbarkeit (in jeder Gruppe nehmen die Zuckungen ab, auch wenn kein sichtbarer Erfolg eingetreten ist.)

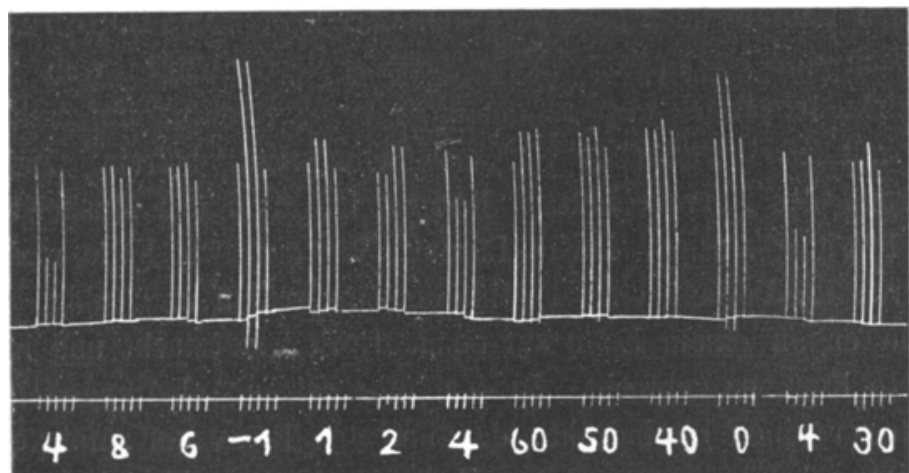

Fig. 4. Versuch 35. Sartorius, vollständig curarisiert. Von rechts nach links zu lesen. Erklärung siehe Fig. 1. Summation bei $0,+1$ und -1 , Subtraktion nur bei 4 , nicht bei 2 und 6 .

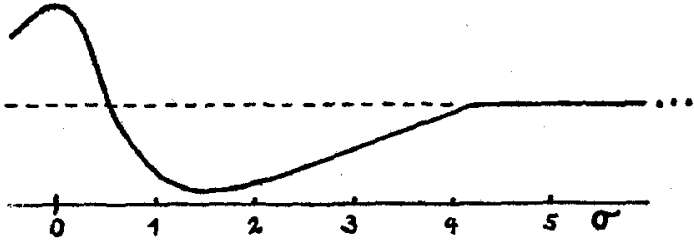

Fig. 5. 9 Quergestreifte Muskeln. Einfluss eines unterschwelligen Reizes auf einen darauffolgenden submaximalen. ..... Zuckungshöhe auf den zweiten -Reiz allein. - Zuckungshöhe auf den Doppelreiz. Die Zahlen bedeuten Intervalle zwischen beiden Reizen; Einheit 1/1000 Sek. (o). 
zu ersehen. Die Kurve ist nach links hin nicht weiter fortgeführt worden, weil der Einfluss eines unterschwelligen Reizes auf einen vorhergehenden submaximalen Reiz bei quergestreiften Muskeln noch nicht untersucht worden ist. Bei glatten Muskeln sind solche Versuche angestellt worden; siehe S. 279 und Fig. 7.

\section{B. Glatte Muskulatur.}

Ausser den beschriebenen Experimenten habe ich noch einige an der Magenmuskulatur des Frosches vorgenommen, um festzustellen, ob auch hier ein Subtraktionsstadium zu finden sei. Bisher kennt man nichts derartiges. R. du Bois-Reymond schreibt bei der Besprechung der glatten Muskeln im Nagel'schen Handbuche ${ }^{1}$ ) „Ein Stadium, in dem sich der Muskel gegen den zweiten Reiz refraktär zejote, existiert also nicht. Ebensowenig kommt der Fall vor, dass die summierte Zuckung geringer ausfiele als die Einzelzuckungen für sich."

Ich richtete mich ganz nach den Vorschriften von P. Schultz ${ }^{2}$ ), die sich gut bewährten. Nahe beim Pylorus wurde vom Magen ein zirkuläres Stück abgetrennt; dann wurde der Ring aufgeschnitten und mit zwei Pinzetten vou der Schleimhaut befreit. Zur Beseitigung des Nerveneinflusses bestrich ich dann den Muskelstreifen mit 1\% iger Atropin-Locke-Lösung; bis auf zwei Fälle wurde er dadurch im Verlauf einiger Minuten ganz schlaff und ruhig. Dann wurde er an einem Ende auf einem Kork festgesteckt und in eine feuchte Kammer gebracht. In der Nähe seiner Anheftungsstelle berübrte er einen Platindraht, der ihm den Reizstrom zuführte; die Ableitung erfolgte am anderen Ende durch einen Platinhaken, von dem aus der Faden zum Schreibhebel gespannt war. Der Muskel wurde also in seiner ganzen Länge durchströmt.

Zur Reizung dienten die beiden grösseren Induktorien mit Kernen, von denen schon bei der allgemeinen Besprechung der Methodik die Rede gewesen ist. Im Vergleich zu den anderen Objekten waren sehr starke Ströme nötig.

Der Plattenträger des Myographions wurde jetzt durch ein Uhrwerk bewegt, so dass er in 1 Sek. $0,042 \mathrm{~mm}$ zurücklegte. Die Ge-

1) Handb. d. Physiol., herausgeg. von W. Nagel Bd. 6 S. 559.

2) P. Schultz, Arch. f. (Anat. u.) Physiol. 1897 S. 307-385; 1908 Suppl. S. $1-134$. 
schwindigkeit betrug also etwa $1 / 100$ der früheren. Im übrigen verliefen die Versuche in derselben Art. Ich musste aber zwischen je zwei Versuchen mehrere Minuten warten, weil der Muskel sich erst nach dieser Zeit vollståndig ausgedehnt hatte. Jede Versuchsserie bestand auch hier aus fünf Teilversuchen, wie S. 272 beschrieben. Daraus kann man ermessen, wie lange eine ganze Versuchsreihe dauerte.

Der sehr leichte Hebel war so belastet, dass er auf den Muskel einen Zug von $1,1 \mathrm{~g}$ ausübte. Er vergrösserte die Bewegungen auf das 16-19 fache.

Die beiden Reize wurden wieder so abgestuft, dass der eine eben unter der Schwelle lag, während der andere mit Sicherheit eine kleine Zuckung hervorrief. Da die Erregbarkeit manchmal schwankte, musste ich hin und wieder die Reizstärke beim Übergang von einem zum anderen Intervall verändern, denn es kam darauf an, den schwächeren Reiz dicht unter der Schwelle zu halten. Dies trug dazu bei, dass meine Ergebnisse nicht so klar sind wie bei quergestreiften Muskeln, und ich spreche daher meine Folgerungen mit einer gewissen Reserve aus.

Das allgemeine Resultat ist, dass ein sicheres Subtraktionsstadium nicht festgestellt werden konnte, wenn der schwächere Reiz v o ra usging. In einigen Fällen war (beim Intervall 0,08 Sek.) eine Andeutung davon zu sehen, die aber vielleicht dem Zufall zuzuschreiben ist (siehe Fig. 6, XIII). Dieser Punkt muss noch mit besserem Versuchsmaterial entschieden werden. Dagegen ist es ganz sicher, das bei dieser Reihenfolge der Reize in der Regel Summation eintritt [beobachtet von $0,042-3,36$ Sek. ${ }^{1}$ )]. Bei Gleichzeitigkeit beider Reize erfolgt, wie nicht anders zu erwarten, Summation. Sehr auffällig ist es aber, dass der Doppelreiz mit grosser Regelmässigkeit weniger wirkt als der stärkere allein, wenn er aus einem submaximalen und einem innerhalb der Latenzzeit desselben nachfolgenden unterschwelligen Stromstoss besteht ${ }^{2}$ ). Das habe ich beobachtet in dem Intervall $-0,042$ bis $-0,252$ Sek. Das Latenzstadium dauerte in allen Fällen mehr als 1 Sek.

Das Gesagte erhellt aus der Tab. 2 und der Fig. 6.

Die graphische Darstellung ergibt die Kurve der Fig. 7.

1) Bei quergestreiften ist es anders, siehe S. 274 oben.

2) Bei quergestreiften Muskeln ist diese Reihenfolge noch nicht untersucht worden. 
Tabelle 2.

Resultate der Doppelreizung von glatten Muskeln. + Summation, - Subtraktion, $=$ Indifferenz. Erster Reiz submaximal, zweiter unterschwellig.

\begin{tabular}{|c|c|c|c|c|c|c|c|c|}
\hline \multirow{2}{*}{ Nr. } & \multicolumn{8}{|c|}{ Intervalle in Millimetern. Einheit $=0,042$ Sek. } \\
\hline & 0 & 1 & 2 & 3 & 4 & 5 & 6 & 7 \\
\hline $\begin{array}{l}\text { VIII } \\
\text { X } \\
\text { XI } \\
\text { XII } \\
\text { IIIX }\end{array}$ & $\begin{array}{l}+ \\
+ \\
+\end{array}$ & $=$ & $\begin{array}{l}- \\
- \\
-\end{array}$ & $=$ & -? & - & - & $=$ \\
\hline
\end{tabular}
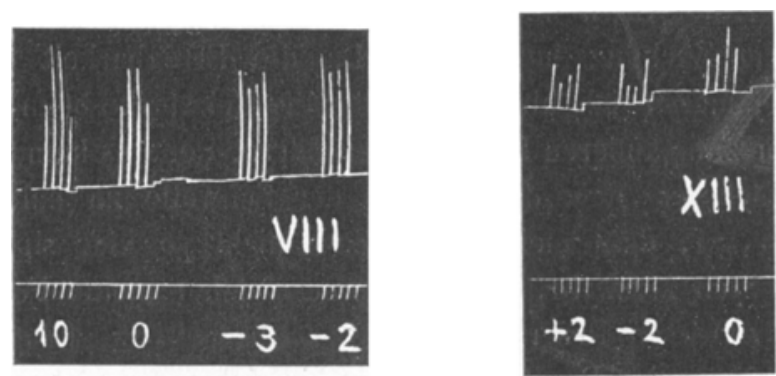

Fig. 6. Versuche VIII und XIII. Atropinisierter Magenring. Von rechts nach lin ks zu lesen. Erklärung siehe Fig. 1, aber Zeiteinheit 0,042 Sek. Negative Intervalle: zuerst der submaximale, dann der unterschwellige Reiz; positive Intervalle umgekehrt. Summation bei 0 und +10 , Subtraktion bei $-3,-2$ und +2 .

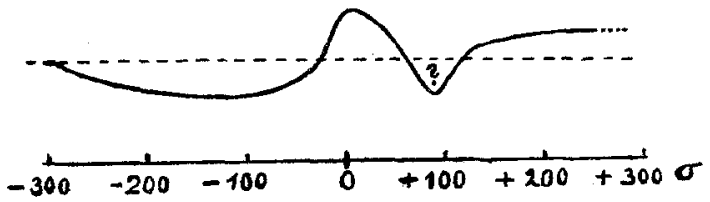

Fig. 7. Glatte Muskeln. Einfluss eines unterschwelligen Reizes auf einen vorhergehenden $(-300$ bis 0$)$ und auf einen folgenden $(0$ bis +300$)$ submaximalen. Die Zahlen bedeuten Intervalle zwischen beiden Reizen; Einheit 1/1000 Sek. $(\sigma)$. .... Zuckungshöhe auf Einzelreizung. - Zuckungshöhe auf Doppelreizung.

\section{Zusammenfassung.}

Im ersten Teile dieser Arbeit wurden quergestreifte Froschmuskeln (Gastrocnemius und Sartorius), teils unvergiftet, teils kurarisiert, durch zwei Öffnungsinduktionsströme direkt gereizt. Der erste Stromstoss wurde so bemessen, dass er dicht unter der Reizschwelle lag, der zweite war submaximal. Das Intervall zwischen beiden Reizen wurde zwischen 0 und 0,0414 Sek. variiert. Bei sehr kleinem Intervall (bis 0,0004 Sek.) verstärken sich die beiden Reize in bezug 
auf die Zuckungshöhe, dann kommt eine Periode der gegenseitigen Abschwächung, und schliesslich ist die Zuckung so hoch, als ob der submaximale Reiz allein gewirkt hätte. Das deckt sich mit den früher von Gildemeister bei indirekter Reizung erhobenen Befunden. Ein direkt oder indirekt unterschwellig gereizter Muskel hat ein nicht sofort einsetzendes, rasch versehwindendes relatives Refraktärstadium, in dem er auf submaximale Reize weniger hohe Zuckungen ausfüht als vorher und nachher.

Im zweiten Teile der Arbeit wurde die glatte Magenmuskulatur des Frosches ebenso untersucht, nachdem sie mit Atropin behandelt worden war. Wenn der untersehwellige Reiz dem submaximalen vora usging, konnte ein Subtraktionsstadium nicht mit Sicherheit festgestellt werden; Andeutungen davon wurden wiederbolt beobachtet. Bei umgekehrter Reihenfolge der Reize aber war ein solches Stadium manchmal deutlich vorhanden, Hier ist die Wirkung eines Doppelreizes vermindert, wenn der unterschwellige Reiz in den Anfang des Latenzstadiums des submaximalen fällt. 